\title{
A Framework for Real Time Processing of Sensor Data in the Cloud
}

\author{
Supun Kamburugamuve, Leif Christiansen, and Geoffrey Fox \\ School of Informatics and Computing and Community Grids Laboratory, Indiana University, Bloomington, IN 47408, USA
}

Correspondence should be addressed to Supun Kamburugamuve; skamburu@indiana.edu

Received 17 November 2014; Accepted 7 April 2015

Academic Editor: Eduard Llobet

Copyright (C) 2015 Supun Kamburugamuve et al. This is an open access article distributed under the Creative Commons Attribution License, which permits unrestricted use, distribution, and reproduction in any medium, provided the original work is properly cited.

\begin{abstract}
We describe IoTCloud, a platform to connect smart devices to cloud services for real time data processing and control. A device connected to IoTCloud can communicate with real time data analysis frameworks deployed in the cloud via messaging. The platform design is scalable in connecting devices as well as transferring and processing data. With IoTCloud, a user can develop real time data processing algorithms in an abstract framework without concern for the underlying details of how the data is distributed and transferred. For this platform, we primarily consider real time robotics applications such as autonomous robot navigation, where there are strict requirements on processing latency and demand for scalable processing. To demonstrate the effectiveness of the system, a robotic application is developed on top of the framework. The system and the robotics application characteristics are measured to show that data processing in central servers is feasible for real time sensor applications.
\end{abstract}

\section{Introduction}

The availability of internet connections and low manufacturing costs have led to a boom in smart objects, devices with a tripartite construction consisting of a CPU, memory storage, and a wireless connection. These smart objects (or devices) are equipped with sensors that produce data and actuators capable of receiving commands. Such devices have proliferated in all fields and their use is expected to grow exponentially in the future. For these devices, central data processing has been shown to be advantageous due to numerous factors, including the ability to easily draw from vast stores of information, efficient allocation of computing resources, and a proclivity for parallelization. Because of these factors, many devices may benefit from processing only some data locally and offloading the remainder to central servers. Among the aforementioned devices, and increasingly present in modern life, are robots. Such examples as the iRobot Roomba, a robot that can clean the floor, present affordable, automated aids for daily living. Additionally, Amazon and Google are researching and developing platforms for delivering consumer products using drones. Most of these robots have limited onboard processing power but still generate large amounts of data. Cloud-based analysis of data from such robots creates many challenges due to strict latency requirements and high volumes of data production.

To process data derived from numerous smart devices, we need scalable data processing platforms. Cloud is an ideal computational platform for hosting data processing applications for smart devices because of its efficiency and agility. Cloud computing [1] refers to both applications delivered as services over the Internet and the hardware and system software in the datacenters that provide those services. Cloud computing enables computing as a utility and is gradually becoming the standard for computation, allowing the systems and users to use Platform as a Service (PaaS), Infrastructure as a Service (IaaS), and Software as a Service (SaaS). The computational nodes are provisioned, configured, and reconfigured dynamically in the cloud and can take the form of virtual machines or physical machines. Furthermore, sensorbased applications can benefit from in-house private cloud environments hosted within organizations or from public clouds hosted by large companies.

In order to process data generated by smart devices in a cloud environment, the data must be transmitted from the devices to the cloud in an efficient and scalable manner. The communication between cloud applications and the devices is essentially based on events, which suggests that 


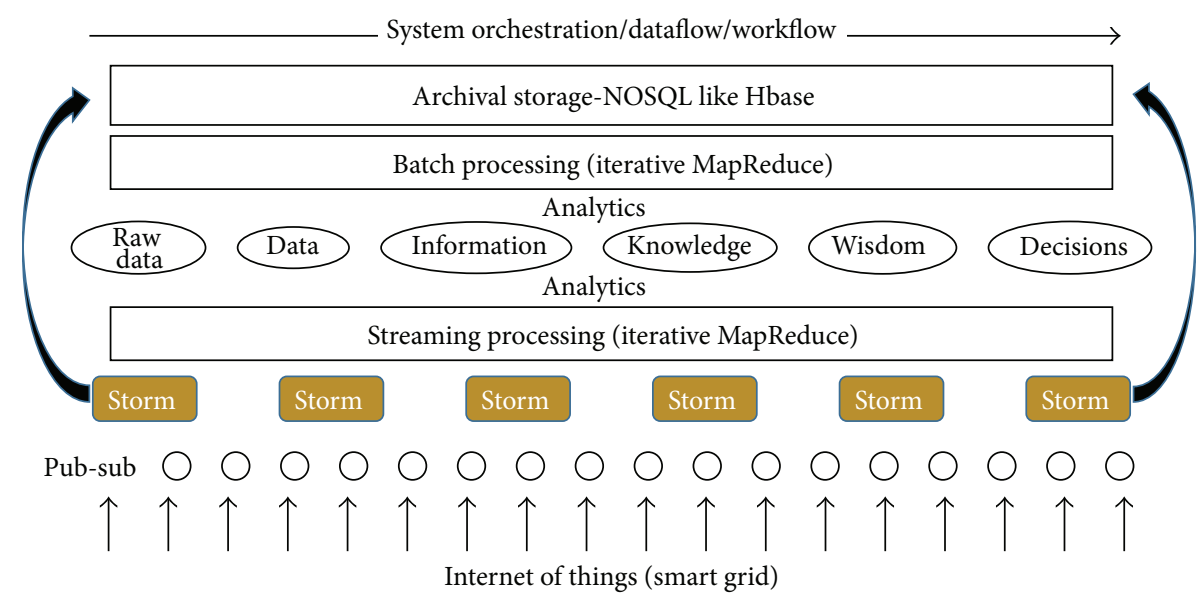

FIgURE 1: DIKW view of the system.

the traditional request/response approach is not appropriate. For example, when using requests and responses, a device requiring real time control has to poll the applications continuously, which increases the latency and network traffic. Transmission of events is well supported by publish-subscribe messaging [2] where a publisher makes information available to subscribers in an asynchronous fashion. Over time, publish-subscribe messaging has emerged as a distributed integration paradigm for deployment of scalable and loosely coupled systems. Subscribers have the ability to express their interest in an event or pattern of events and are subsequently notified of any event generated by a publisher which matches their registered interest. An event is asynchronously propagated to all subscribers that registered interest. Publishsubscribe messaging decouples the message producers and consumers in the dimensions of time, space, and synchronization. The decoupling favors the scalability of the message producing and consuming systems. Because of these features, publish-subscribe messaging is potentially a good fit for connecting smart devices to cloud applications.

Two widely used schemes of pub-sub systems are topicbased and content-based. In topic-based systems, the messages are published to topics which are identified by keywords. The consumers subscribe to and receive messages coming to these topics. In content-based systems, the consumers subscribe to messages based on the properties of the messages. This means the content of each message has to be examined at the middleware to select a consumer among possibly a large set of consumers. Because of the simple design of most topic-based middleware, they tend to scale well compared to content-based brokers and introduce less overhead.

We can assume that, for all our devices, data is sent to a cloud as a stream of events. It is important to process the data as a stream before storing it to achieve real time processing guarantees. Parallel processing of events coming from a single source can help to reduce the latency in most applications. The ability to connect large numbers of devices creates a need for a scalable infrastructure to process the data. Distributed event processing engines (DSPEs) [3-6] are a good fit for such requirements. A DSPE abstracts out the event delivery, propagation, and processing semantics and greatly simplifies the real time algorithm development. They also act as a messaging fabric that distributes data for batch processing and archival purposes to other data sinks like databases and file systems after some preprocessing of the data.

We envision a cloud-based data-intensive computing architecture where stream-based real time analysis and batch analysis are combined together to form a rich infrastructure for sensor applications. We propose Cloud DIKW- (Data, Information, Knowledge, and Wisdom-) based architecture for sensor data analysis in the cloud. The high level DIKW view of the system is shown in Figure 1. With DIKW architecture, the data enters the processing pipeline through the DSPE layer. Both stream analysis and batch analysis are combined to continuously evolve the data models to transition from raw data to decisions. The storage layer acts as the glue between the batch analysis and the stream analysis.

By combining the above requirements, we have developed our IoTCloud platform, which is a distributed software platform capable of connecting devices to the cloud services. IoTCloud uses topic-based publish-subscribe messaging to transfer data between the devices and the cloud services and a DSPE to process the data in the cloud. The platform supports two publish-subscribe brokers with different semantics that are suitable for different applications. We have developed a robotic application that runs through a private in-house cloud to demonstrate how to use the system and measured the characteristics of the system. Doing so demonstrates that we can achieve real time processing of sensor data in a cloud environment in a scalable manner. The main contribution of our work is to explore scalable cloud-based real time data processing for sensor applications.

Section 2 of the paper describes the related work in this area. Section 3 explains the architecture of the framework and Section 4 highlights the robotics application we have developed. In Section 5, we present a series of experiments done to evaluate the system and discuss the resulting observations. Finally, in Sections 6 and 7, we end with conclusions and future work. 


\section{Related Work}

Hassan et al. [7] presented a content-based publish/subscribe framework for connecting sensor data to cloud services. Content-based pub-sub allows greater flexibility for the application designers than topic-based systems. But content-based setups usually involve higher overhead because the brokers have to inspect message content. Furthermore, content-based pub-sub brokers are neither popular nor widely available as products.

Mires [8], TinySIP [9], and DV/DRP [10] are all publish/ subscribe messaging middleware for Wireless Sensor Networks (WSNs). They address the different issues in connecting WSNs and communicating with sensors. MQTT-S [11] is an open topic-based pub-sub protocol defined for transferring data from sensors. The protocol enables data transfer between sensors and traditional networks. In our work, we assume that sensor data is available to be transported to cloud services and we handle the transfer of gathered data from devices to cloud services. For example, a device connected to our system can send data via a dedicated communication channel, public Internet, and so forth. Also, many devices can be connected in WSNs using the above-mentioned protocols or messaging systems after which our platform can transfer this data to cloud services for processing.

Reference architectures for integrating sensors and cloud services have been discussed in the literature $[12,13]$. Both works explore the general architecture that can be used to connect sensors to cloud services and the potential issues. In our work, we provide a framework that can be used to send sensor data from devices to the cloud as well as showing how to process the data within a generic framework. We also discuss how to transfer data and process it in a scalable way, topics that are not fully addressed in the above papers. A detailed survey of some of the existing work done on cloud robotics has been summarized in [14]. Our framework can be used as a generic platform for developing cloud robotics applications such as collective robot learning, robot swarms, and robot perception based on image processing.

\section{IoTCloud Architecture}

A system view of the architecture is shown in Figure 2. Our architecture consists of three main layers:

(1) Gateway layer.

(2) Publish-subscribe messaging layer.

(3) Cloud-based big data processing layer.

We consider a device as a set of sensors and actuators. Users develop a driver that can communicate with the device and deploy it in a gateway. This driver does not always have to directly connect to the device. For example, it can connect via a TCP connection or through a message broker. The data generated by the driver application is sent to the cloud-processing layer using publish-subscribe messaging brokers. The cloud-processing layer processes the data and sends control messages via the message brokers back to the driver, which converts the information to a format that suits

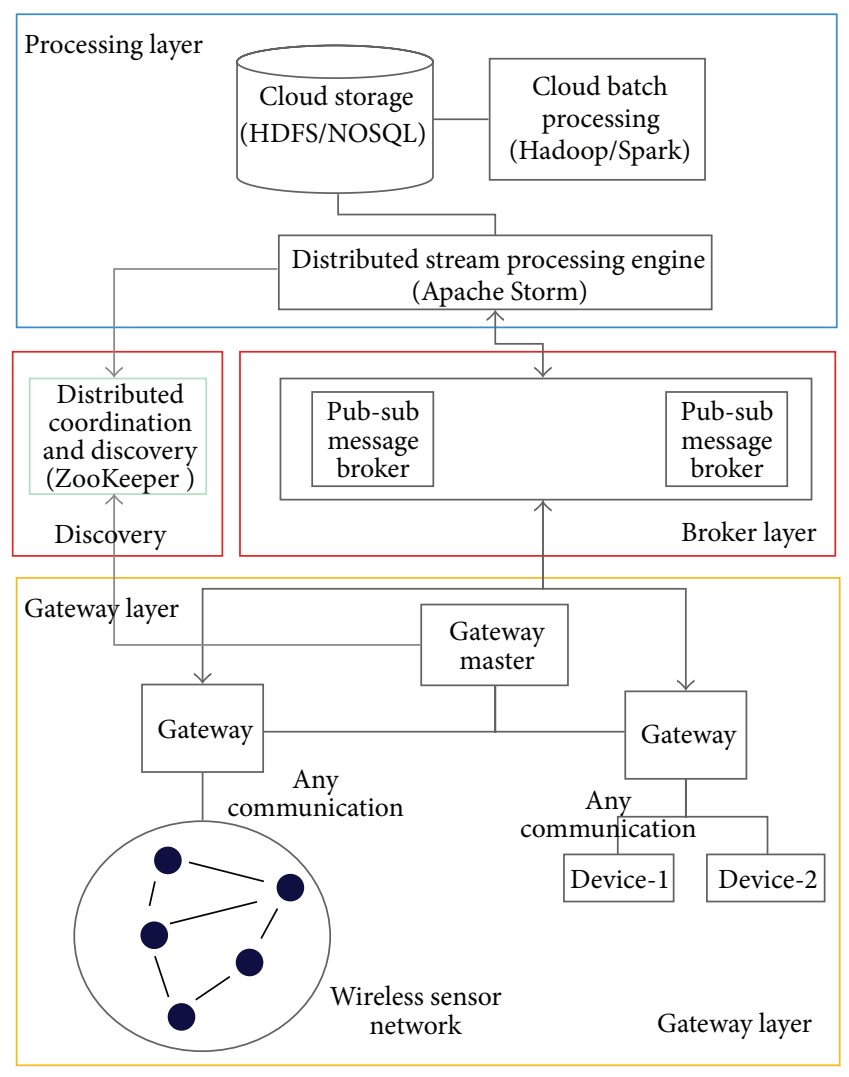

FIGURE 2: IoTCloud architecture.

the device and communicates this back to it. The platform is implemented in Java programming language.

3.1. Gateway. Drivers are deployed in gateways responsible for managing drivers. There can be multiple gateways in the system and each has a unique ID. A gateway master controls the gateways by issuing commands that include deploy/ undeploy and start/stop drivers. A gateway is connected to multiple message brokers, which can be in a cluster configuration. By default, the platform supports RabbitMQ [15], ActiveMQ, and Kafka [16] message brokers. Gateways manage the connections to the brokers and handle the load balancing of the device data to the brokers. They update the master about the drivers deployed in it and the status of the gateways. The master then stores the state information in a ZooKeeper [17] cluster.

3.2. Driver. The driver is the data bridge between a device and the cloud applications. It serves to convert data coming from the device into a format that the cloud applications expect and vice versa. A driver has a name and a set of communication channels. When a driver is deployed, the running instance gets an instance ID. This is used for controlling the driver after the deployment. The same driver can be deployed multiple times and each of the instances receives a unique ID. One driver can have multiple communication channels each with a unique name. A communication channel connects the driver to publish-subscribe messaging brokers. When 
a driver is deployed, its information is saved in ZooKeeper. The default structure of driver information in ZooKeeper is

\section{/iot/sensors/[driver_name]/ [driver_instance_id] / [channel_name]}

A ZooKeeper node (ZNode) with the driver instance ID contains information about the driver such as its status and metadata. ZNodes with a channel name contain information about the channels. The framework allows shared and exclusive channels to be created. An exclusive channel can give faster communication between the drivers and the cloud processing. But in large-scale deployment of drivers, an exclusive channel can result in a large number of resources in the brokers. Some applications do not have strict latency requirements and can use shared channels, thus consuming less system resources.

3.3. Brokers. The platform specifically focuses on topic-based publish-subscribe brokers rather than content-based models. We chose topic-based brokers for several reasons: (1) stable, open-source topic-based brokers are easily available; (2) topic-based brokers are simple to use and configure; (3) the overhead introduced by the broker is minimal compared to content-based versions. For this project, the most important factors are 1 and 3, because our applications require low latency and topic-based brokers are the ones readily available for use. The messaging layer needs to preserve the message ordering, preventing multiple consumers from consuming messages off the same driver.

There are many open-source brokers available that fulfill our needs for the messaging infrastructure. Such brokers include ActiveMQ [18], RabbitMQ [15], Kafka [16, 19] Kestrel, and HonertMQ. From these, ActiveMQ, RabbitMQ, and Kafka are widely used topic-based publish-subscribe brokers. The preliminary studies show that ActiveMQ and RabbitMQ have identical functionalities for our purposes but the latter is capable of handling more load with less overhead. The Kafka broker has very good clustering capabilities and can handle parallel consumer reads for the same topic. For these reasons, we decided to support both RabbitMQ and Kafka in our platform.

Each communication channel created in a driver is connected with a topic created in the message broker. The framework supports two mappings of channels to topics, thus creating two types of channels. In the first type, each channel is mapped to a unique queue in the broker. We call this type exclusive channels. In the other type, a set of channels shares the same topic in the broker and is called a shared channel. At the moment, we use a very simple rule to map the channels to a shared queue. We map the same channel from multiple instances of a driver deployed in one gateway to a single topic:

For shared channels, number of topics $=$ number of gateways.

Forexclusive channels, number of topics $=$ number of driver instances.

For a shared channel, the corresponding topic name is of the format "gateway_id.driver_name.queue_name". For an exclusive channel, it is "gateway_id.driver_name.driver_id .queue_name”.

RabbitMQ. RabbitMQ is a message broker primarily supporting Advanced Message Queuing Protocol (AMQP) [20]. Even though the core of RabbitMQ is designed to support AMQP, the broker has been extended to support other message protocols like STOMP, MQTT, and so forth. RabbitMQ is written in Erlang programing language and supports low latency high throughput messaging. It has a rich API and architecture for developing consumers and publishers; plus topics are easy to create and manage using its APIs. These topics are lightweight and can be created without much burden to the broker. We allow both shared channels and exclusive channels to be created for RabbitMQ. The metadata of a message is sent using RabbitMQ message headers and includes sensor ID, gateway ID, and custom properties.

Kafka. Kafka is a publish-subscribe message broker backed by a commit log. The messages sent by the producers are appended to a commit log and the consumers read the messages from this. Kafka implements its own message protocol and does not support standard protocols like AMQP or MQTT. At the core of Kafka messaging is the concept of a topic. A topic is divided into multiple partitions and a message is sent to a single partition. In our platform, the partition for a message is chosen using a key accompanying a message. Thus, messages with the same key go to the same partition. Consumers consume messages from partitions. Partitions of a single topic can spread across a cluster of Kafka servers. Furthermore, a single partition is replicated in a Kafka cluster for reliability. Kafka guarantees ordering of messages in a partition and does not guarantee ordering across partitions. Because a topic consists of multiple partitions, consumers can read from the same topic in parallel without affecting the message ordering for a single message key. In IoTCloud platform, we use the driver ID as the key for a message.

IoTCloud needs to send metadata with a message, such as the driver ID, site ID, and custom properties. Because Kafka only supports byte messages without any headers, we use a Thrift- [21] based message format to send metadata about the message. Use of driver ID as the key ensures that the messages belonging to a single driver instance will always be in one partition. We use at most one consumer per partition to ensure the message ordering for a driver. Because Kafka topics can be partitioned, we will have parallel read-and-write capabilities for shared channels. Because of this, the platform only supports shared channels for Kafka.

3.4. Cloud Processing. As the primary cloud-processing framework, we use Apache Storm [6], which is an opensource DSPE. There are many DSPEs available but we chose Storm because of its scalability, performance, excellent development community support, and its ability to use scripting languages to write applications. Storm can be used to process the data and send responses back immediately, or it can do some preprocessing of the data and store it for later processing by batch engines such as Apache Hadoop. The applications we have developed do not use batch processing at 
the moment, so we have not incorporated such engines into the platform yet. But our architecture permits integration of engines like Hadoop. We use FutureGrid [22] as our cloud platform for deploying the Storm Cluster since it has an OpenStack installation and we can provision VM images using the OpenStack tools.

Apache Storm. Storm is a distributed stream processing engine designed to process large amounts of streaming data in a scalable and efficient way. Data processing applications are written as Storm topologies. A topology defines a DAG structure for processing the streaming data coming from the devices as an unbounded stream of tuples. The DAG consists of a set of spouts and bolts written to process the data. The tuples of the stream flow through the nodes (spouts and bolts) of the DAG. Spouts and bolts are primarily written in Java but other programming languages like Python and Ruby are permitted. Data enters a topology through spouts and the processing happens in bolts. The components in the DAG are connected to each other using stream (tuple) groupings. Pub-sub is a common pattern for ingesting data into a Storm topology. A bolt can consume the connected input streams, do some processing on the tuples, and generate and emit new tuples to the output streams. Usually, the last bolts in the topology DAG write the results to a DB or send the results to remote nodes using pub-sub messaging. The spouts and bolts of a topology can be run in parallel in different computation nodes.

To ease the development of Storm topologies in our platform, we allow the external communication points of a Storm topology to be defined in a configuration file. Below is one such example configuration. The topology has two external communication channels. A "kinect_receive" spout gets the input data from devices and a "count_send" bolt sends output information back to the devices. We can use this configuration to build the outer layer of a topology automatically. The algorithm has to be written by the application developer

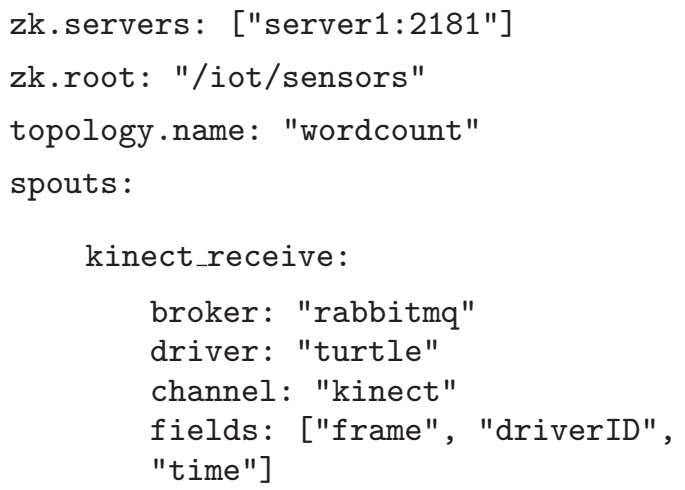

bolts :

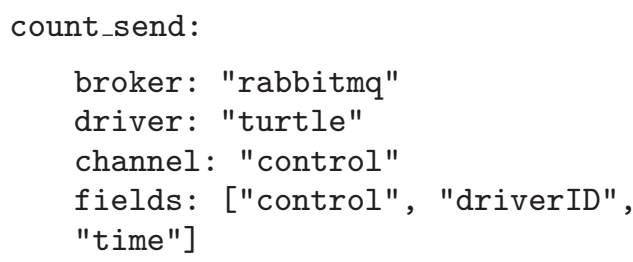

We can run many instances of any of the components in a Storm topology in parallel. For example, to read data in parallel from many devices, we can spawn several instances of the kinect_receive spout in different nodes. This can be done for any bolt in the topology as well. The parallelism can be changed at runtime as well. This allows the system to scale with the addition of drivers.

3.5. Discovery. Because Storm is a distributed processing framework, it requires coordination among the processing units. For example, when a communication channel is created in the broker for a device, the parallel units responsible for communicating with that channel should pick a leader because multiple units reading from the same channel can lead to data duplication and out of order processing, which is not desirable for most applications. Also, the distributed processing units should be able to detect when the drivers come online and go offline. To adapt to such a distributed dynamic processing environment, we need discovery and coordination. Apache ZooKeeper [17] can achieve both. When drivers come online, the information about them is saved in ZooKeeper. The discovery component discovers and connects this information to the cloud processors dynamically at runtime. This allows the processing layers to automatically distribute the load and adjust accordingly to the changes in the data producer side.

When a topology deploys its external communication components (spouts and bolts), it does not know about the physical addresses of the topics or how many topics it has to listen to. So, at the very beginning, the topology does not have any active message listeners or senders. The topology has information about the ZooKeeper and the drivers that it is interested in. It uses this information to dynamically discover the topics that it has to listen to and add those consumers and producers to the topology at runtime.

3.6. Processing Parallelism. The processing parallelism at the endpoints of the topology is bound to the message brokers and how we can distribute the topics across the brokers. For processing bolts at the middle, maximum parallelism is not bounded and depends on the application. A Storm topology gets its messages through the spouts. The same spout can run multiple instances in parallel to read the messages coming from multiple devices connected to the system. A spout always reads the messages from a single channel of a device. If a processing algorithm requires input from multiple channels, the topology must have multiple spouts. A running instance of a spout can connect to multiple topics to read the messages, but all these topics must be connected to a channel with the same name and driver. When a spout needs to read from multiple topics, the topology distributes the topics equally among the running instances of the spout dynamically at runtime. The message flow through the Storm topology happens primarily using the driver IDs. The bolts that are communicating with the brokers know about all the topics in the system and they can send a message to an appropriate topic using the driver ID.

RabbitMQ. There is a limit to the number of parallel spouts that we can run due to the number of topics created per 


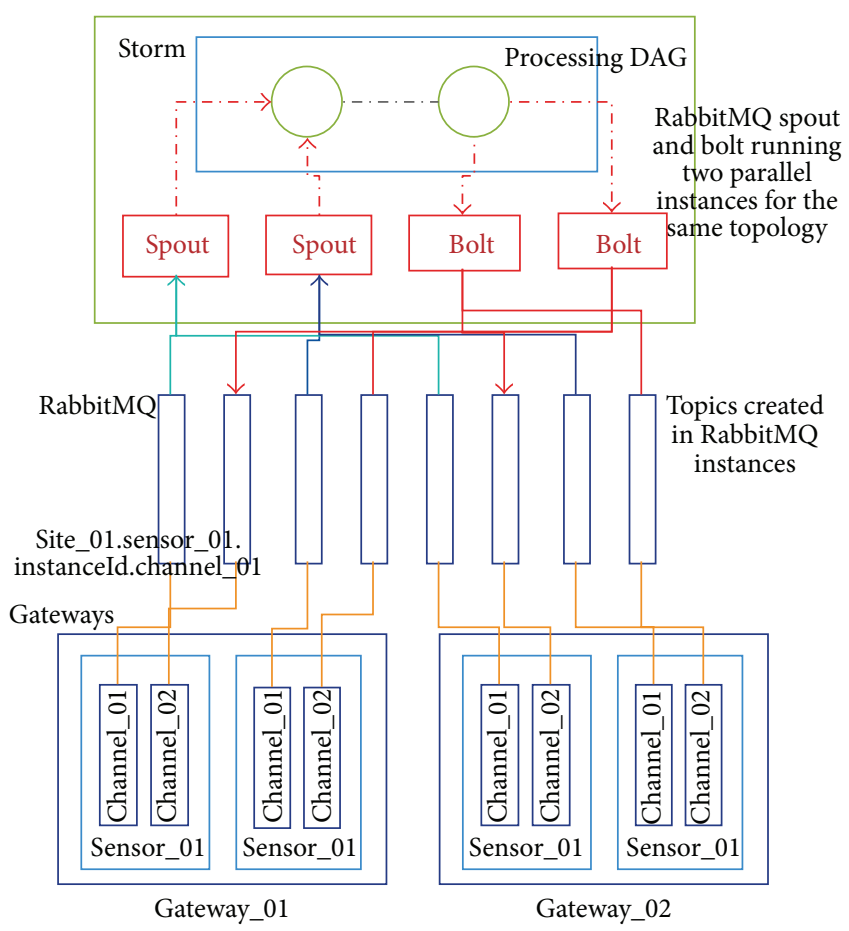

FIgURE 3: RabbitMQ exclusive channels and Storm.

channel. The following gives an upper bound on how many spouts we can run when RabbitMQ brokers are used:

Shared channels: number of parallel spouts $\leq$ number of gateways.

Exclusive channels: number of parallel spouts $\leq$ number of channels.

In general, we cannot do parallel reads from a topic due to the ordering constraints. Figure 3 shows how exclusive channels created by a driver named sensor_01 are connected to the Storm topology. Here, the Storm topology runs only one instance for each spout reading from channel_01 and channel_02. Because we have 8 channels in 4 instances of the drivers, we need 8 topics in the broker. Since we only have 2 spouts and 2 bolts in the topology, each spout is connected to 2 topics and each bolt is communicating with 2 topics. Figure 4 shows the same scenario with shared channels. In this case, we only have 4 topics because the two drivers deployed in the same gateway are using the same topics.

Kafka. Kafka topics are more heavyweight than RabbitMQ. For every topic in the system, Kafka has to create log files and index files in the file system for its partitions. If the replication is enabled for fault tolerance, these files have to be replicated in the Kafka cluster. Kafka also supports parallel reads for a single topic. Because of these reasons, we only support shared channels for Kafka, where the number of spouts possible depends on the number of partitions for a topic:

number of parellel spouts

$\leq$ number of gateways $\times$ partitions per topic.

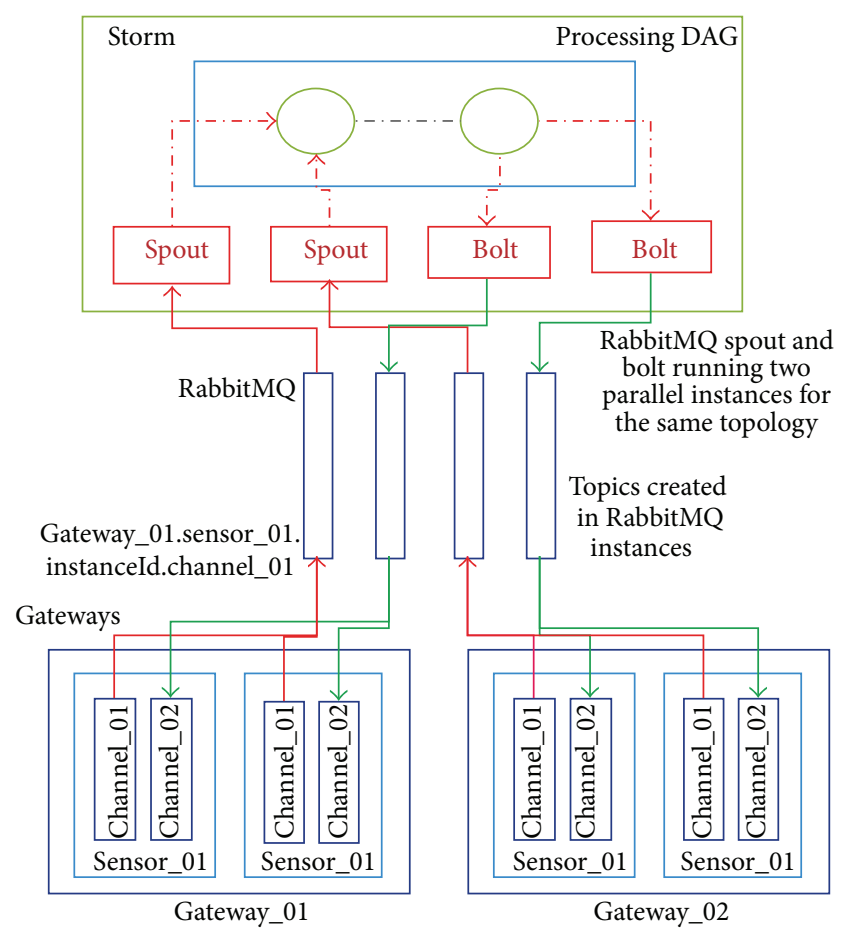

FIgURE 4: RabbitMQ shared channels and Storm.

Figure 5 shows topics distribution with Kafka for the same scenario as in Figure 3. In Figure 5, each Kafka topic has 2 partitions and we have 4 topics because the channels are shared. Read-and-write parallelism in this case is equal to the exclusive channel scenario with RabbitMQ (Figure 4) since each topic has two partitions. But in practical scenarios, we will have fewer partitions than devices connected per gateway. This will make the parallelism greater than the shared channels with RabbitMQ but less than the exclusive channels.

\section{TurtleBot Follower Application}

In order to explore possible configurations for the IoTCloud framework, we used Microsoft Kinect [23] and TurtleBot [24]. Microsoft Kinect consists of an IR camera, an RGB camera, an IR emitter, and several auxiliary features. Our project was not concerned with the details of the hardware but complete discussions of the Kinect specifications and method of depth calculation are available. Currently, there are numerous open-source projects and academic studies utilizing Kinect due to the sensor's affordability and host of applications. In addition, a well-documented robot incorporating Kinect is already available: TurtleBot by Willow Garage (see Figure 6). For these reasons, they were chosen as a subject for the development of a sensor to cloud-processing framework.

In our application, TurtleBot follows a large target in front of it by trying to maintain a constant distance to the target. Compressed depth images of the Kinect camera are sent to the cloud and the processing topology calculates command messages, in the form of velocity vectors, in order to maintain a set distance from the large object in front of TurtleBot. These 


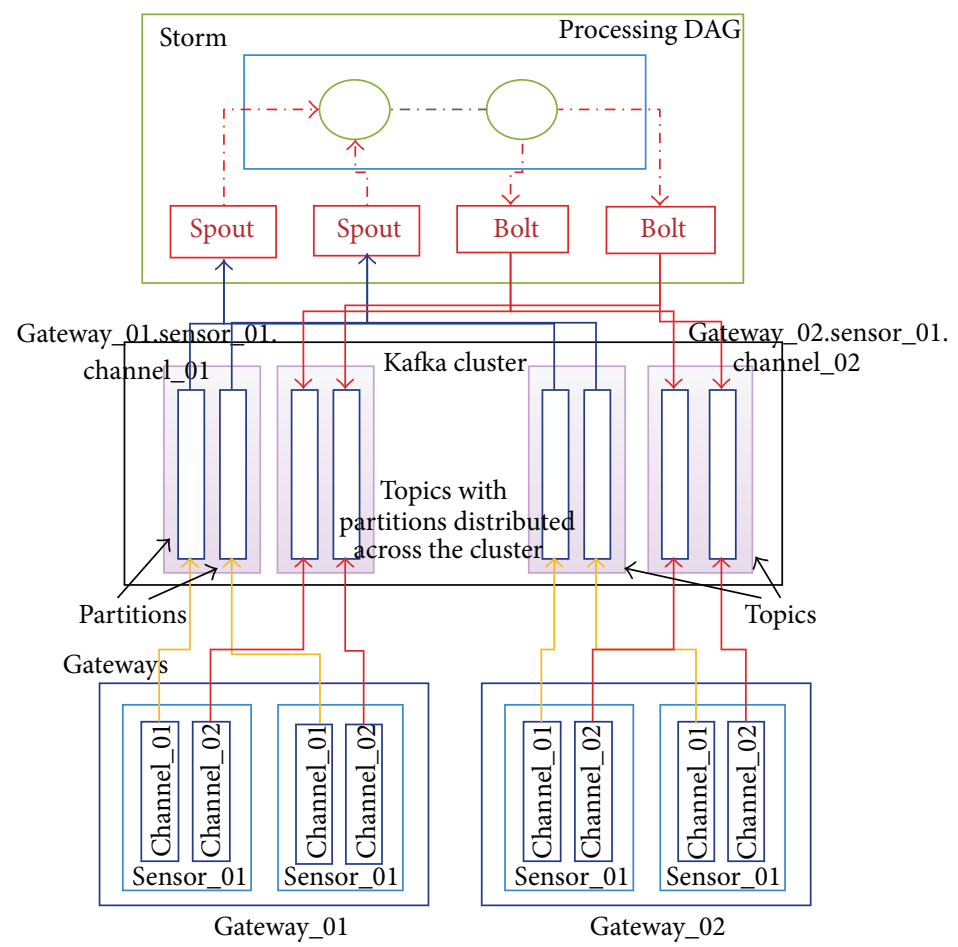

FIGURE 5: Kafka shared channels and Storm.

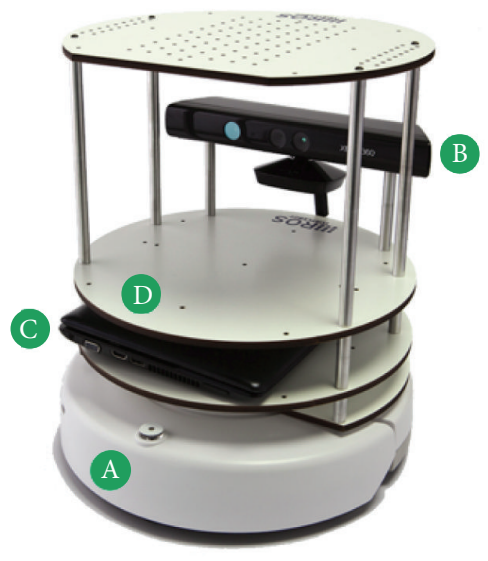

(A) Mobile base and power board

(B) 3D sensor

(C) Computing

(D) TurtleBot hardware

Figure 6: TurtleBot.

command messages are sent back to TurtleBot using its ROS [25] API. TurtleBot then actuates these vectors to move.

4.1. Reading Depth Frames from Kinect. The initial step in developing our application utilizing the Kinect depth camera was finding a driver to read in the Kinect data stream. TurtleBot is operated with ROS, the open-source robotics operating system, which has an available Kinect driver.
The ROS Kinect driver is built on OpenKinect's libfreenect [26] driver, so, in order to avoid any unnecessary overhead, libfreenect was used. Libfreenect is an open-source Kinect driver that provides a Java interface to both the IR and the RGB cameras. Methods are provided to start a depth stream and handle frames. Libfreenect was originally implemented in $\mathrm{C}++$, although a Java JNA wrapper is now available.

4.2. Compression. During the course of the project, several compression schemes were tested. In the early stages, this included the LZ4, Snappy [27], and JZlib Java compression libraries. Snappy achieved less compression but was faster than the other two. Ultimately, we chose a two-stage compression process using the Mehrotra et al. [28] inversion technique as the first stage and Snappy as the second. The Mehrotra et al. [28] inversion technique takes advantage of the error endemic to the depth camera. The depth camera's accuracy decreases proportional to the inverse of the squared depth. Hence, multiple values may be encoded to the same number without any loss in fidelity [28]. By using this inversion technique, every two-byte disparity can be compressed to one byte. It is worth noting, however, that the inversion algorithm takes distance as an input, not disparity. Mehrotra et al. achieved a startling $5 \mathrm{~ms}$ compression time for their whole 3-step process with little optimization. For the sake of expediency, our project used an existing Java compression library (Snappy) rather than the Mehrotra et al. RLE/Golomb-Rice compression.

The last major decision left was whether to implement the prediction strategy mentioned in Mehrotra et al. This strategy takes advantage of the heterogeneous nature of the depth of 
objects. This translates into long runs of values in the depth data. The prediction strategy is simple and converts any run into a run of 0s. For an RLE, this will have a clear advantage, but when tested with Snappy the gain was negligible and thus not worth the added computation. Ultimately, we were able to achieve a compression ratio of $10: 1$ in a time of $10 \mathrm{~ms}$. This compares favorably to the $7: 1$ ratio in $5 \mathrm{~ms}$ reached by Mehrotra et al. The data compression happens in the laptop computer inside TurtleBot. After compression, the data is sent to a driver application that runs in an IoTCloud gateway. This gateway relays the information to the cloud.

4.3. Calculation of Velocity. The Storm topology for this application consists of 3 processing units arranged one after the other. First spout receives the compressed Kinect frames; next bolt uncompresses this data and calculates the velocity vector required by TurtleBot to move. The algorithm running in this bolt calculates a point cloud of TurtleBot's field of view using an approximation technique mentioned in [29]. Then, it uses the point cloud to calculate an average point, the centroid, of a hypothetical box in front of TurtleBot. Shifts in the centroid are calculated and command messages, in the form of vectors, are generated. Last bolt sends these vectors to TurtleBot.

All the literature indicates that the Kinect should stream each depth frame as 307,200 11-bit disparity values, 2047 being sent to indicate an unreadable point. But upon inspection of received disparity values, the highest value observed was 1024. When this value was treated as the unreadable flag, the depth map displayed appeared normal. Depth shadows were rendered correctly along with the minimum and maximum readable distances. The code was then adjusted to expect only 10-bit disparity values, after which everything functioned normally. The full range of the Kinect, $80 \mathrm{~cm}-400 \mathrm{~cm}$, can be encoded with only 10-bit values. It is unclear whether the 10-bit values are a result of Java libfreenect wrapper or faulty code, but our programs are fully functional and the issue was left unresolved. An explanation of this phenomenon would no doubt prove beneficial and may be a point of latter investigation.

4.4. Controlling TurtleBot. The driver running in the gateway receives the velocity vectors from the application in the cloud. It then converts these vectors to a format that the ROS API of TurtleBot accepts. Ultimately, the ROS API is used by the driver to control TurtleBot. We use a Java version of ROS available for interfacing with ROS, which is primarily written in Python.

\section{Results and Discussion}

We mainly focused on the latency and the scalability of the system. A series of experiments were conducted to measure latency and how well the system performs under deployment of multiple sensors. We used FutureGrid as our cloud platform and deployed the setup on FutureGrid OpenStack medium flavors. An instance of medium flavor has 2 VCPUs, $4 \mathrm{~GB}$ of memory, and $40 \mathrm{~GB}$ of hard disk. We ran Storm Nimbus and ZooKeeper on 1 node, gateway servers on 2

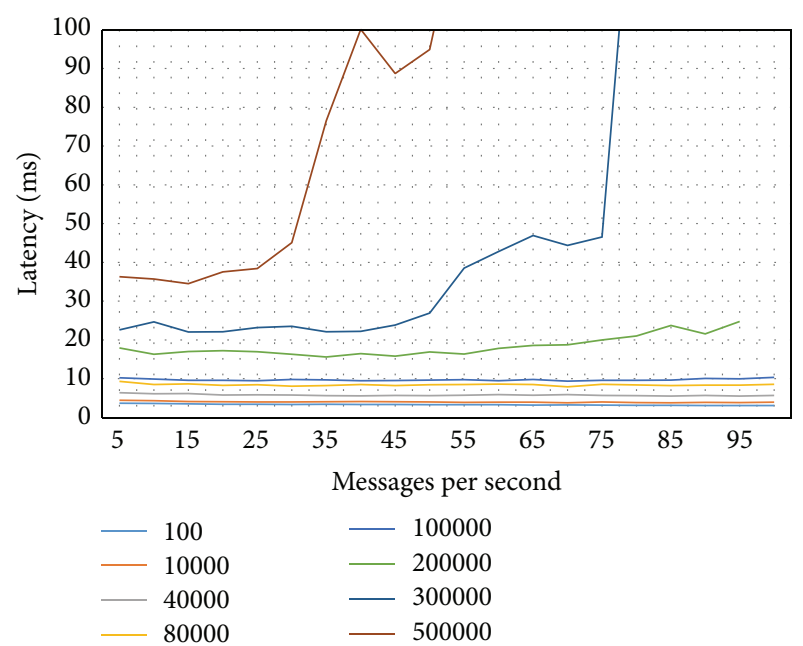

FIGURE 7: Average latency for different message sizes with RabbitMQ. The different lines are for different message sizes in bytes.

nodes, Storm supervisors on 3 nodes, and brokers on 2 nodes. Altogether, our setup contained 8 virtual machines with moderate configurations.

To test the latency of the system, we deployed 4 driver applications on the two gateways that produce data at a constant rate. This data was relayed through the two brokers and injected into a Storm topology, which passed the data back to the gateways. The topology was running 4 spout instances in parallel to get the data and 4 bolts in parallel to send the data out. The round-trip latency was measured at the gateways for each message. This setup was repeated for different message sizes and message rates. We went up to 100 messages per second and increased the messages size up to $1 \mathrm{MB}$. Each driver sent 200 messages and we recorded the average across all the drivers. We tested the system with RabbitMQ and Kafka brokers. For measuring the scalability, we progressively increased the number of drivers deployed in the gateways and observed how many devices can be handled by the system.

TurtleBot application is an application deployed on FutureGrid. We observed that TurtleBot was able to follow a human in front of it when this application was deployed. We tested TurtleBot application through the Indiana University computer network and measured the latency observed.

5.1. Latency. Figure 7 shows the latency observed when running the tests through a RabbitMQ server. Up to $200 \mathrm{~KB}$ messages, the latency was at a considerably lower value for all the message rates we tested. At $300 \mathrm{~KB}$ messages, the latency started to grow rapidly after a message rate of 50 was reached.

Figure 8 shows the average latency observed with the Kafka broker. We noticed some drastically high latency values, and when the size of the messages increases beyond $40 \mathrm{~K}$, these variations became frequent. The frequency of these values increased the average latency considerably. The increase in latency can be attributed to the fact that Kafka brokers are designed to be run in machines with high disk I/O rates and our tests were done on computation nodes that do not have very good I/O performance. There are other 


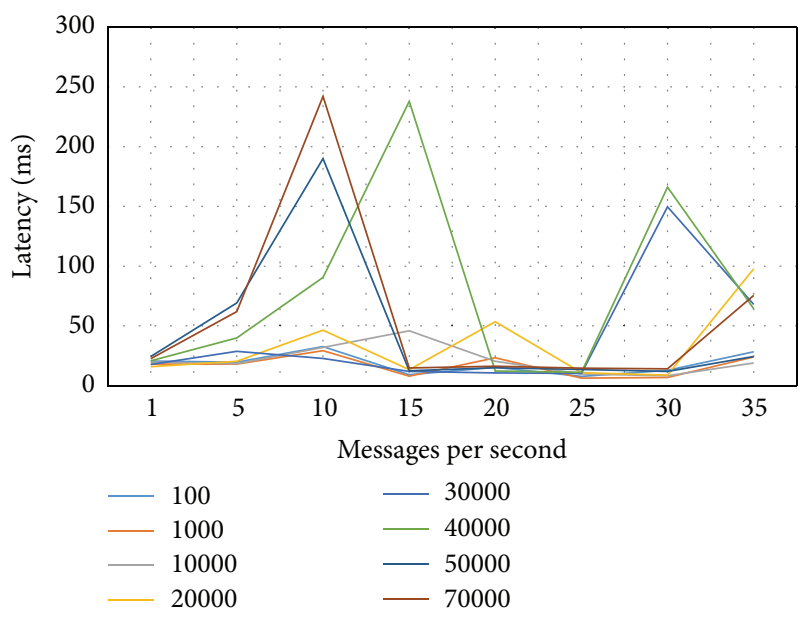

FIGURE 8: Average latency for different message sizes with Kafka. The different lines are for different message sizes in bytes.

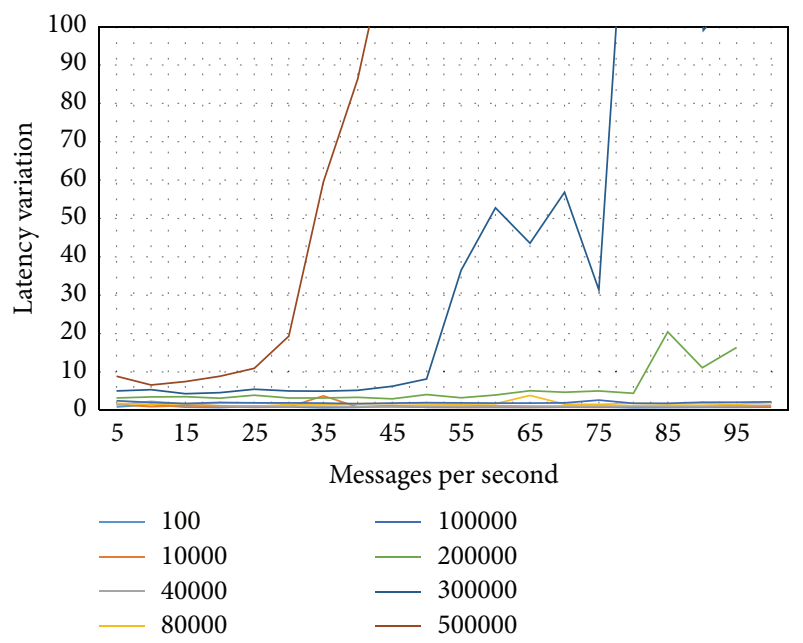

Figure 9: Latency standard deviation with different message sizes and message rates for RabbitMQ. The different lines are for different message sizes in bytes.

performance results of Kafka that were done on high disk I/O nodes that show some large variations in latency as well [30]. Despite variations in latency, on average the system was running with a considerably low latency using Kafka. In our setup, Kafka broker latency began to increase much more quickly than RabbitMQ brokers. We have reported these issues to the Kafka development community. Kafka is a relatively new project under development and we believe its development community is working on fixing these issues in future versions.

5.2. Jitter. For most real time applications, uniformity of the latency over time is very important. Figure 9 shows the latency variation in observed latencies for a particular message size and rate with RabbitMQ broker. The variation was also minimal for message sizes up to $200 \mathrm{~KB}$. After that,

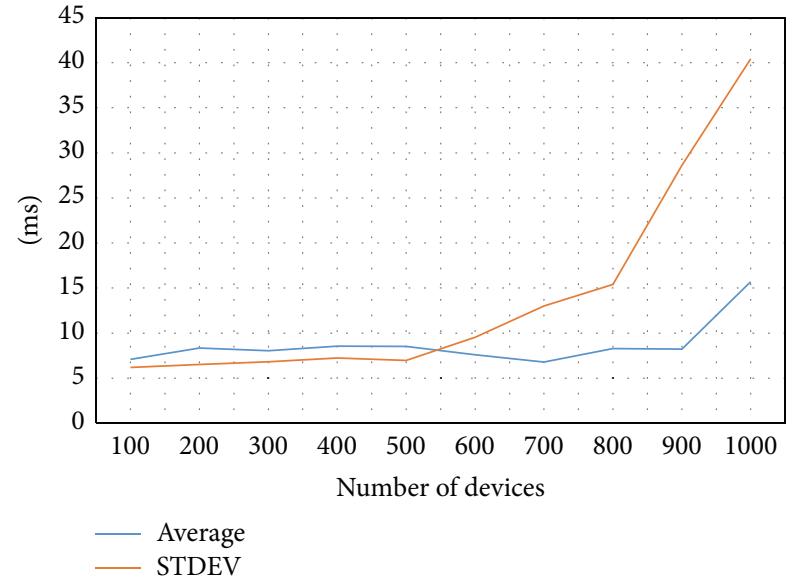

FIGURE 10: Latency with varying number of devices, RabbitMQ. The average latency and standard deviation are shown.

there was a large variation in the latency. The Kafka latency variation is very high compared to RabbitMQ broker and we are not including those results here.

5.3. Scalability. In the test we did for observing the scalability of the system, we deployed 1000 mock drivers in two gateways and measured the latency. These drivers can generate 100-byte messages at a rate of 5 messages per second. We used low values for both message rate and message size so that we could make sure the system did not slow down due to the large amount of data produced. Figure 10 shows the latency with RabbitMQ. Latency observed was marginally higher than the previous test we did with 4 drivers, but it was consistent up to 1000 drivers and stayed within reasonable range. The increase in latency can be attributed to increased use of resources. At 1000 sensors, the latency started to increase. Because this test was done in shared channel mode, only 2 spouts were actively reading from the 2 queues created.

We performed the same test with the Kafka broker. Because we partitioned each topic into 4 , all 4 spouts were actively reading from the topics. This is the advantage of having a Kafka-like distributed broker. The latency observed is shown in Figure 11. As expected, there were large variations observed. We tried to remove these big numbers and draw the graph to see how they affect the average latency. Figure 11 shows graphs with values $>200$ removed. We can observe that the average latency is at a considerable low range after these very high values are removed.

All the tests were done for the best case scenario in terms of latency of Storm-based analysis. A real application would involve much more complex processing and a complicated DAG structure for data processing. Those processing latencies will add to the overall latency in real applications. Also in our tests we sent and received the same message through the cloud. In real applications, messages generated after the processing are usually minimal compared to the data messages, so we expect a reduction in latency as well. 


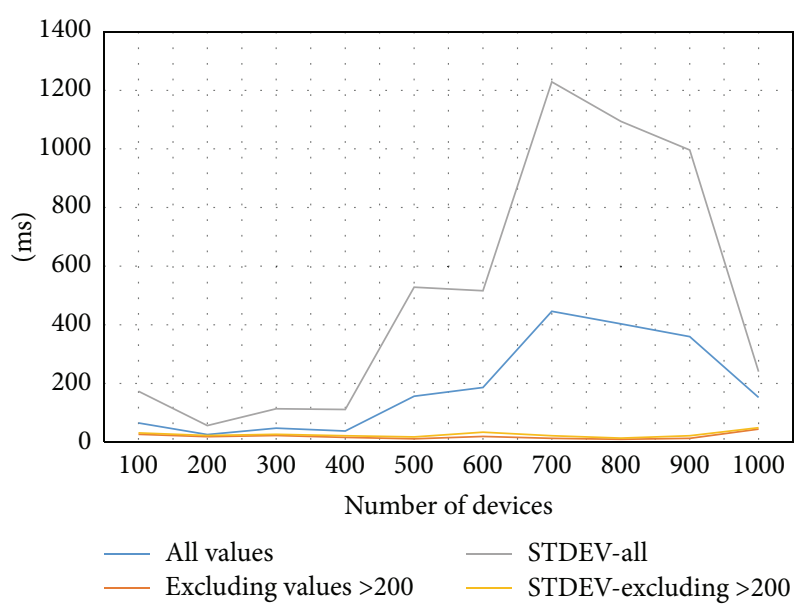

Figure 11: Latency with varying number of devices, Kafka. The average latency and standard deviation are shown. Also, averages calculated with omitting values over 200 are shown.

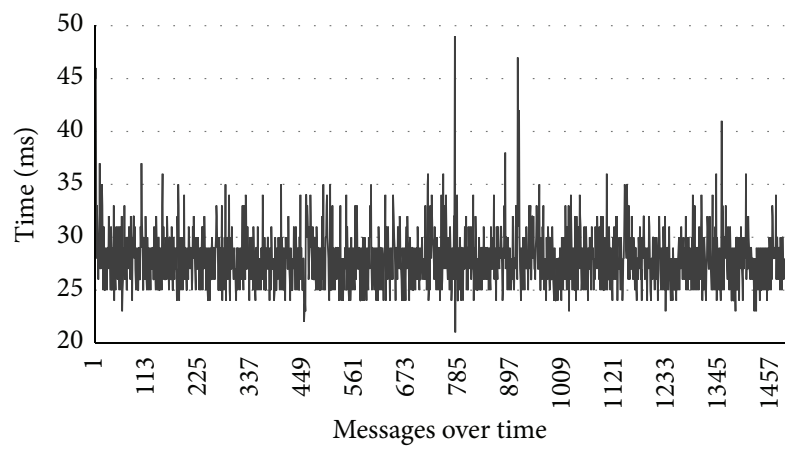

FIGURE 12: Latency observed in TurtleBot application.

5.4. TurtleBot. Because of the latency requirements, we used the RabbitMQ broker for TurtleBot application. TurtleBot was functioning properly under the latencies we have observed. Figure 12 shows the latency values we observed for 1500 Kinect frames. The average latency fluctuated between $35 \mathrm{~ms}$ and $25 \mathrm{~ms}$. TurtleBot was sending messages of size $60 \mathrm{~KB}$ in a 20 -message/sec rate. The best case latency without any processing for such messages is around $10 \mathrm{~ms}$. The network latency and the processing add another $25 \mathrm{~ms}$ to the latency. The processing includes both compression and decompression time of Kinect frames. There were some outliers that went to values such as $50 \mathrm{~ms}$. These were not frequent but can be seen occurring with some high probability. We could not recognize any patterns in such high latency observations; some explanations for these increases might be network congestion, Java garbage collection, and other users employing the same network and resources in FutureGrid. We observed average latency of 33.26 milliseconds and standard deviation of 2.91 .

\section{Conclusions}

In this paper, we introduced a scalable, distributed architecture for connecting devices to cloud services and processing data in real time. Further, we discussed a robotics application built on top of this framework. We investigated how to scale the system with topic-based publish-subscribe messaging brokers and a distributed stream processing engine in the cloud. We measured the performance characteristics of the system and showed that we can achieve low latencies with moderate hardware in the cloud. Also, the results indicate we can scale the architecture to hundreds of connected devices. Because of the low latencies, RabbitMQ broker is suitable for applications with real time requirements. Applications involving massive amounts of devices without strict latency requirements can benefit from the scalability of Kafka brokers. The results also indicate that reasonably uniform behavior in message processing latencies can be maintained, which is an important factor for modeling most problems.

\section{Future Work}

As our platform evolves, we would like to extend our system to Cloud DIKW applications, which involve both real time analysis and batch analysis. A primary concern for real time applications is the recovery from faults. A robot guided by a cloud application should work amidst application level failures and middleware level failures. We would like to explore different fault tolerance techniques for making our platform more robust. The discovery of devices is coarse-grained at the moment and we hope to enable finer-grained discovery of devices at the cloud-processing layer. For example, selecting devices that meet specific criteria like geographical locations for processing is important for some applications. We observed that there are variations in the latency observed in our applications. In some applications, it is required to contain the processing latency with hard limits. It will be interesting to look at methods for enabling such guarantees for our applications. Simultaneously, we are working to build new robotics applications based on our platform.

\section{Conflict of Interests}

The authors declare that there is no conflict of interests regarding the publication of this paper.

\section{Acknowledgments}

The authors would like to thank the Indiana University FutureGrid team for their support in setting up the system in FutureGrid NSF Award OCI-0910812. This work was partially supported by AFOSR Award FA9550-13-1-0225 "Cloud-Based Perception and Control of Sensor Nets and Robot Swarms."

\section{References}

[1] M. Armbrust, A. Fox, R. Griffith et al., "A view of cloud computing," Communications of the ACM, vol. 53, no. 4, pp. 50$58,2010$.

[2] P. T. Eugster, P. A. Felber, R. Guerraoui, and A.-M. Kermarrec, "The many faces of publish/subscribe," ACM Computing Surveys (CSUR), vol. 35, no. 2, pp. 114-131, 2003. 
[3] D. J. Abadi, Y. Ahmad, M. Balazinska et al., "The design of the borealis stream processing engine," in Proceedings of the Conference on Innovative Data Systems Research (CIDR '05), pp. 277-289, Asilomar, Calif, USA, January 2005.

[4] B. Gedik, H. Andrade, K.-L. Wu, P. S. Yu, and M. Doo, "SPADE: the system s declarative stream processing engine," in Proceedings of the ACM SIGMOD International Conference on Management of Data (SIGMOD '08), pp. 1123-1134, June 2008.

[5] L. Neumeyer, B. Robbins, A. Nair, and A. Kesari, "S4: Distributed stream computing platform," in Proceedings of the 10th IEEE International Conference on Data Mining Workshops (ICDMW '10), pp. 170-177, Sydney, Australia, December 2010.

[6] Q. Anderson, Storm Real-Time Processing Cookbook, Packt, 2013.

[7] M. M. Hassan, B. Song, and E.-N. Huh, "A framework of sensor-cloud integration opportunities and challenges," in Proceedings of the 3rd International Conference on Ubiquitous Information Management and Communication (ICUIMC '09), pp. 618-626, January 2009.

[8] E. Souto, G. Guimarães, G. Vasconcelos et al., "Mires: a publish/subscribe middleware for sensor networks," Personal and Ubiquitous Computing, vol. 10, no. 1, pp. 37-44, 2006.

[9] S. Krishnamurthy, "TinySIP: providing seamless access to sensor-based services," in Proceedings of the 3rd Annual International Conference on Mobile and Ubiquitous Systems: Networking and Services (MobiQuitous '06), pp. 1-9, San Jose, Calif, USA, July 2006.

[10] C. P. Hall, A. Carzaniga, and A. L. Wolf, "DV/DRP: a contentbased networking protocol for sensor networks," Tech. Rep. 2006/04, Faculty of Informatics, University of Lugano, Lugano, Switzerland, 2006.

[11] U. Hunkeler, H. L. Truong, and A. Stanford-Clark, "MQTT-Sa publish/subscribe protocol for wireless sensor networks," in Proceedings of the 3rd IEEE/Create-Net International Conference on Communication System Software and Middleware (COMSWARE '08), pp. 791-798, January 2008.

[12] S. K. Dash, J. P. Sahoo, S. Mohapatra, and S. P. Pati, "Sensorcloud: assimilation of wireless sensor network and the cloud," in Advances in Computer Science and Information Technology. Networks and Communications, pp. 455-464, Springer, 2012.

[13] A. Alamri, W. S. Ansari, M. M. Hassan, M. S. Hossain, A. Alelaiwi, and M. A. Hossain, "A survey on sensor-cloud: architecture, applications, and approaches," International Journal of Distributed Sensor Networks, vol. 2013, Article ID 917923, 18 pages, 2013.

[14] B. Kehoe, S. Patil, P. Abbeel, and K. Goldberg, "A survey of research on cloud robotics and automation," IEEE Transactions on Automation Science and Engineering, vol. 12, no. 2, pp. 398409, 2015.

[15] A. Videla and J. J. Williams, RabbitMQ in Action, Manning, 2012.

[16] J. Kreps, N. Narkhede, and J. Rao, "Kafka: a distributed messaging system for log processing," in Proceedings of the 6th International Workshop on Networking Meets Databases (NetDB '11), Athens, Greece, 2011.

[17] P. Hunt, M. Konar, F. P. Junqueira, and B. Reed, "ZooKeeper: wait-free coordination for internet-scale systems," in Proceedings of the USENIX Conference on USENIX Annual Technical Conference (USENIXATC '10), p. 9, USENIX Association, Boston, Mass, USA, June 2010.

[18] B. Snyder, D. Bosnanac, and R. Davies, ActiveMQ in Action, Manning Publications, 2011.
[19] K. Goodhope, J. Koshy, J. Kreps et al., “Building LinkedIn's realtime activity data pipeline," IEEE Data Engineering Bulletin, vol. 35, no. 2, pp. 33-45, 2012.

[20] S. Vinoski, "Advanced message queuing protocol," IEEE Internet Computing, vol. 10, no. 6, pp. 87-89, 2006.

[21] A. Agarwal, M. Slee, and M. Kwiatkowski, "Thrift: scalable cross-language services implementation,” Tech. Rep., Facebook, 2007, http://thrift.apache.org/static/files/thrift-20070401.pdf.

[22] G. Fox, G. von Laszewski, J. Diaz et al., "FutureGrid-a reconfigurable testbed for Cloud, HPC and grid computing," in Contemporary High Performance Computing: From Petascale toward Exascale, Computational Science, Chapman \& Hall, CRC Press, 2013.

[23] Z. Zhang, "Microsoft kinect sensor and its effect," IEEE Multimedia, vol. 19, no. 2, pp. 4-10, 2012.

[24] TurtleBot, 2014, http://wiki.ros.org/Robots/TurtleBot.

[25] M. Quigley, K. Conley, B. Gerkey et al., "ROS: an open-source robot operating system," in Proceedings of the ICRA Workshop on Open Source Software, p. 5, Kobe, Japan, May 2009.

[26] Openkinect, “Open Kinect,” 2014, http://openkinect.org/.

[27] Google, "snappy," 2014, https://code.google.com/p/snappy/.

[28] S. Mehrotra, Z. Zhang, Q. Cai, C. Zhang, and P. A. Chou, "Lowcomplexity, near-lossless coding of depth maps from kinectlike depth cameras," in Proceedings of the 3rd IEEE International Workshop on Multimedia Signal Processing (MMSP '11), pp. 1-6, November 2011.

[29] Openkinect, "Imaging Information," http://openkinect.org/ wiki/Imaging_Information.

[30] P. Kozikowski, "Kafka 0.8 Producer Performance," http://liveramp.com/blog/kafka-0-8-producer-performance-2/. 

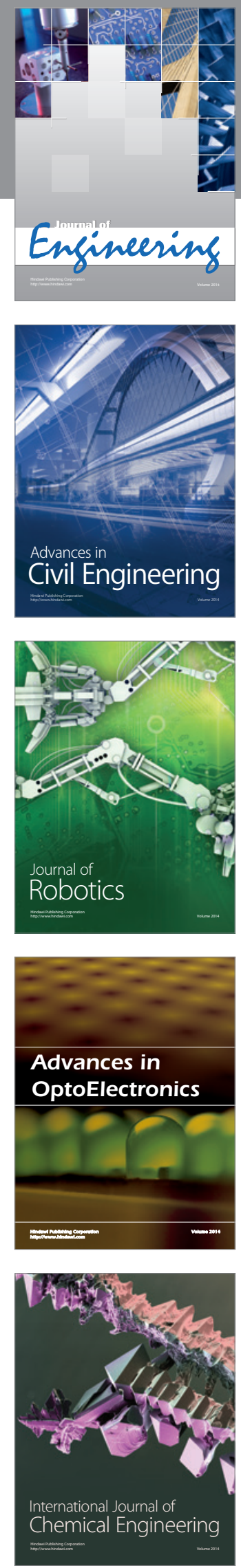

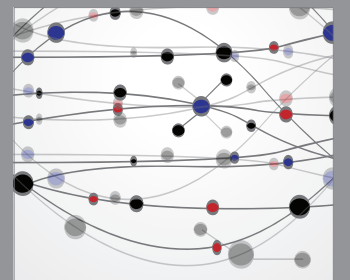

The Scientific World Journal
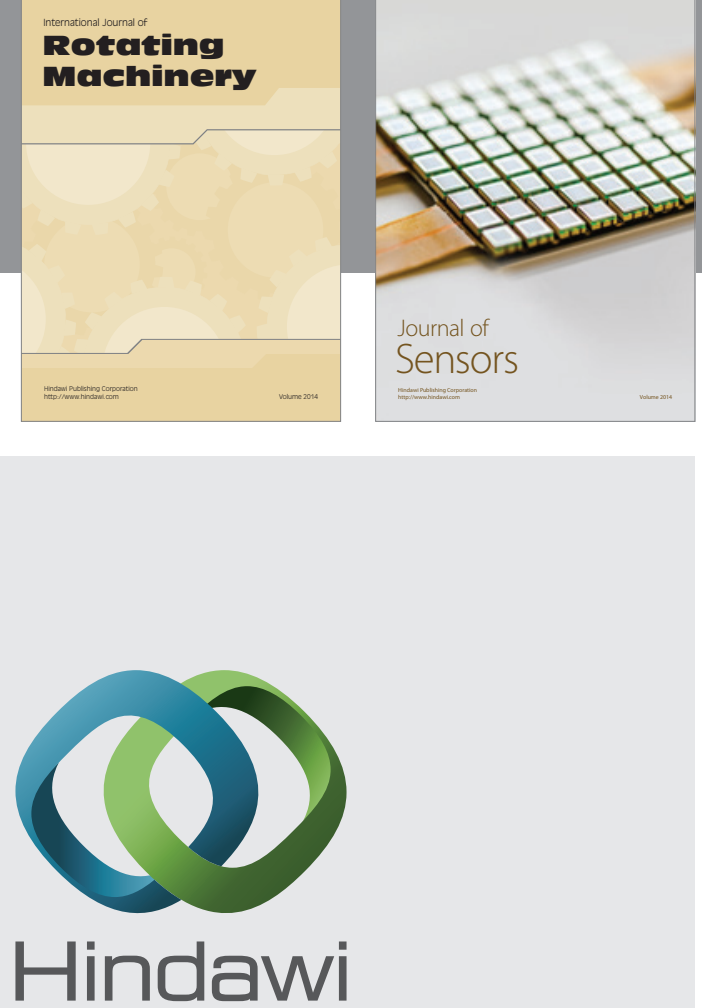

Submit your manuscripts at http://www.hindawi.com
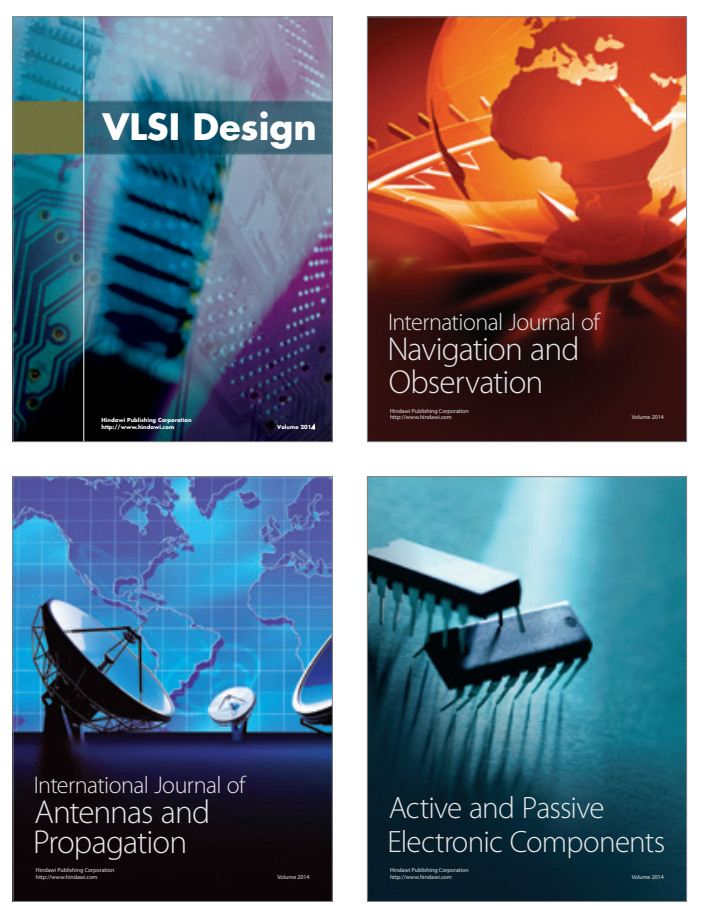
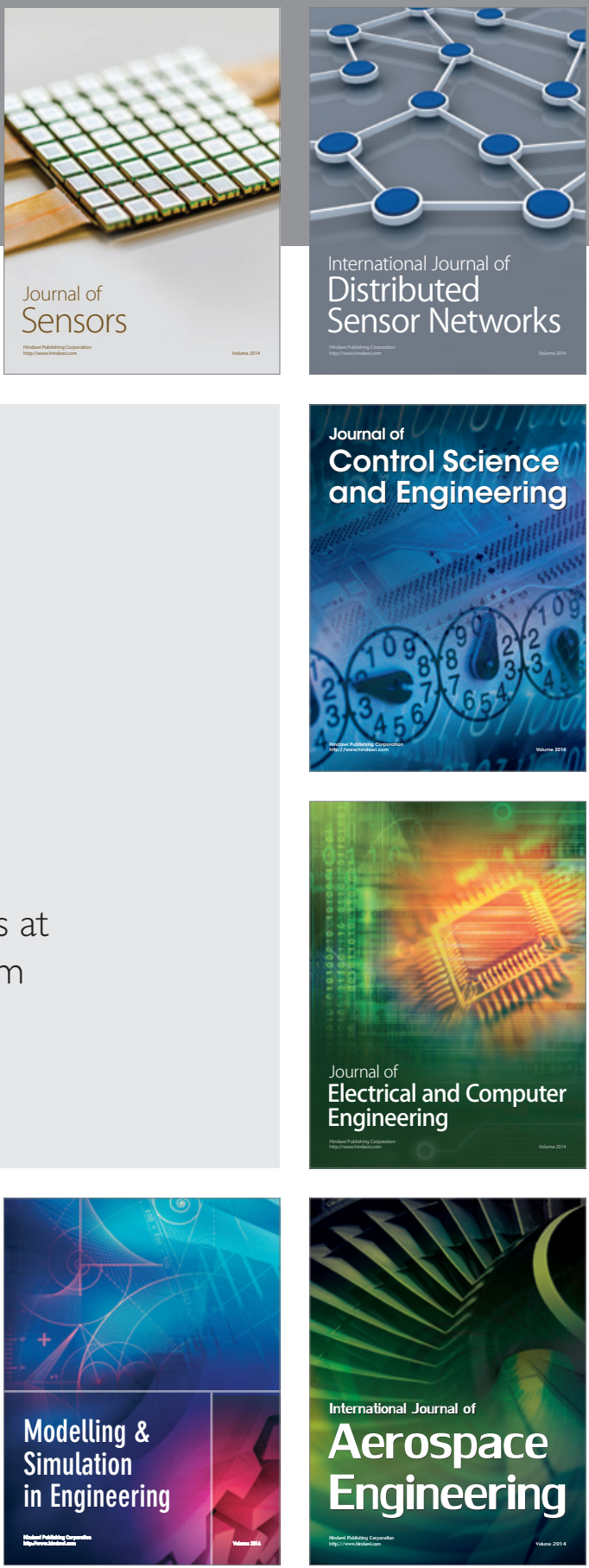

Journal of

Control Science

and Engineering
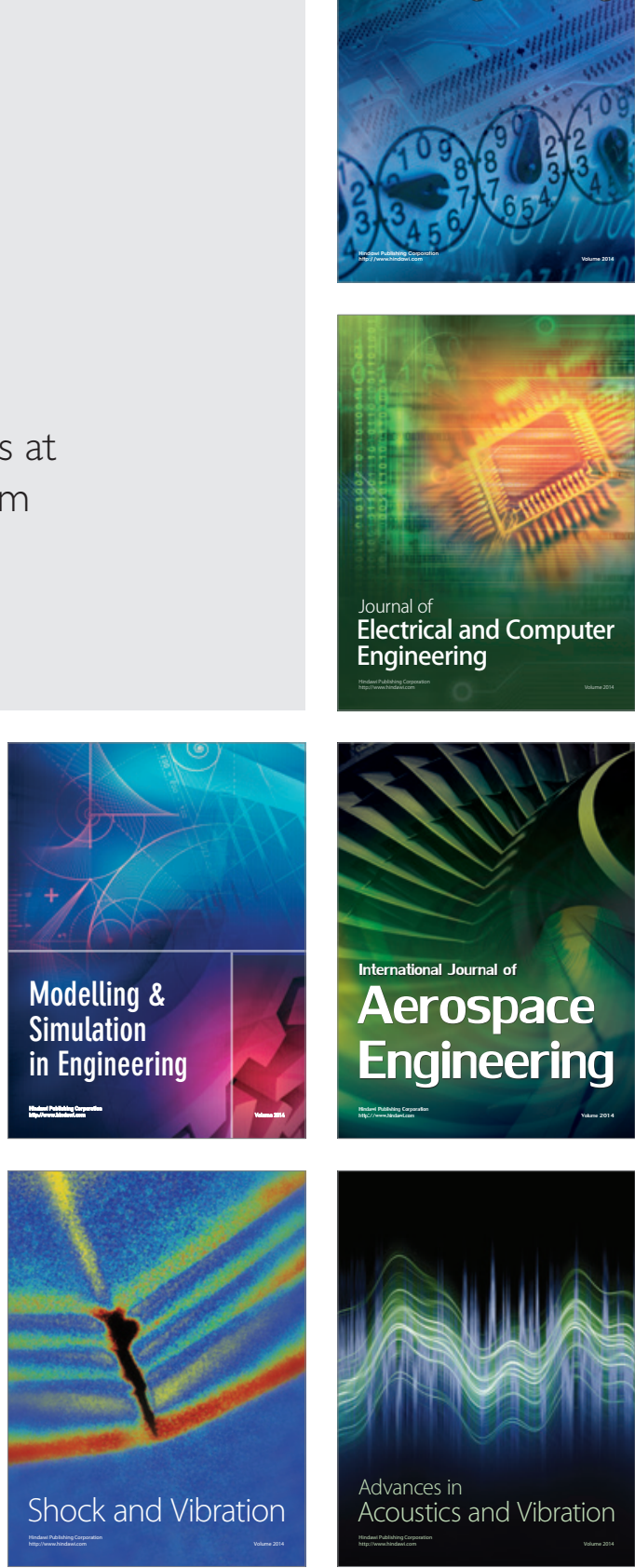